\title{
Spatial and temporal characterization of fish assemblages in a tropical coastal system influenced by freshwater inputs: northwestern Yucatan peninsula
}

\author{
Daniel Arceo-Carranza \& Ma. Eugenia Vega-Cendejas \\ CINVESTAV-IPN, Unidad Mérida, Km. 6 Antig. Carr. a Progreso, A. P. 73 Cordemex, C.P. 97310 Mérida, Yucatán, \\ México; darceo@mda.cinvestav.mx; maruvega@mda.cinvestav.mx
}

Received 06-VII-2007. Corrected 10-VII-2008. Accepted 14-VIII-2008.

\begin{abstract}
Coastal lagoons are important systems for freshwater, estuarine and marine organisms; they are considered important zones of reproduction, nursery and feeding for many fish species. The present study investigates the fish assemblages of the natural reserve of Dzilam and their relationship with the hydrologic variables. A total of 6474 individuals (81 species) were collected, contributing with more than $50 \%$ considering the Importance Value Index (IVI), Sphoeroides testudineus, Fundulus persimilis, Anchoa mitchilli, Eucinostomus gula, Eucinostomus argenteus and Mugil trichodon. Differences in species composition were found between seasons the highest during the cold fronts. Spatially, differences were related with the presence of freshwater seeps, the highest in the ecological characterized eastern part and the lowest with higher difference in specific composition located in the western part of the internal zone, due to a higher abundance and dominance of $L$. rhomboides. Salinity and temperature were the variables that presented a higher influence in the distribution of some pelagic species such as A. mitchilli and A. hepsetus. Because of the abundant freshwater seeps characteristic of the coastal lagoons of Yucatan Peninsula their community structure and fish assemblage display spatial and temporal differences in specific composition. Rev. Biol. Trop. 57 (1-2): 89-103. Epub 2009 June 30.
\end{abstract}

Key words: Ichthyofauna, species composition, temporal variation, freshwater inputs, environmental variables, Bocas de Dzilam.

Coastal lagoons and estuaries are environments featured by being transitional places between land and marine habitats, as well as dynamic systems with highly environmental variability in short and long term. So that, their biological communities are influenced mainly by physical environment (Kupschus and Tremain 2001), and their structure, diversity and stability are defined by biologic interactions (intra and interspecific) (Francis et al. 2002). Within these ecosystems, fish constitute the main biological component of marine and estuarine species using them as feeding, reproduction, growth and protection grounds (Raz-Guzmán and Huidobro 2002), in terms of their tolerance limits to those hydrologic conditions (Whitfield 1999). Variables affecting their distribution in estuarine waters are salinity, temperature, turbidity, and dissolved oxygen, though sediment type heterogeneity and vegetation, also influence the availability of nutritional resources and the protection against predators (Blaber and Blaber 1980, Marchand 1993, Blaber 1997, Marshall and Elliot 1998). Estuarine fish assemblages show cyclical variations in abundance and composition, and since they include species of fresh and marine waters, they can show sedentary or migratory behaviors and be present in diverse stages of their development (Simier et al. 2004) as well as seasonal changes in their habitat types, because of coastal processes. 
Few studies have been realized in Yucatan Peninsula and specifically the ichthyofauna community in "Bocas de Dzilam" Reserve has not been assessed (Vega-Cendejas et al. 1993, Vega-Cendejas et al. 1994, Vega-Cendejas et al. 220). Because of its reserve status and to its ecological importance for estuarine ichthyofauna, this research aims to investigate fish assemblages distribution related to climatic season and some hydrologic variables.

Study area. "Bocas de Dzilam" lagoon declared a protected natural area since 1989 (Zone of Ecological Conservation, Reserve of Dzilam), is located in Southeastern Mexico in the central coast of Yucatan State $\left(21^{\circ} 19^{\prime}-21^{\circ}\right.$ $32^{\prime} \mathrm{N}, 88^{\circ} 35^{\prime}-88^{\circ} 58^{\prime} \mathrm{W}$ ) with a surface of $9.4 \mathrm{~km}^{2}, 12.9 \mathrm{~km}$ long, a maximum width of $1.65 \mathrm{~km}$ with a permanent connection with the sea (375 $\mathrm{m}$ wide), bordered by mangrove, and depth $1-2.5 \mathrm{~m}$, with $80 \%$ of the bottom covered by macrophytes, dominated mainly by Halodule wrightii and Ruppia maritima (Herrera-Silveira et al. 1998, Medina-Gómez and Herrera-Silveira 2003). It is featured by the presence of subterranean flows and springs giving it a special nature by the presence of ecotones regulated by fresh and salty water.

\section{MATERIALS AND METHODS}

Six bimonthly samplings including fish and physical variables were performed from May 2003 to March 2004 during climate seasons of rain (July -September), northern cold fronts "norths" (November - January) and dry (May - March) along 8 stations distributed in different environments. Fish sampling was realized with a beach seine (15 m x $1.5 \mathrm{~m} ; 2.5$ $\mathrm{cm}$ mesh size), hauls by duplication covering an average area of $240 \mathrm{~m}^{2}$. Prior collection, physicochemical variables were measured in situ (temperature, salinity and dissolved oxygen) using a Yellow Springs Instrument (model $85)$. Fish were preserved in $10 \%$ formalin, identified, counted, and measured for standard length to the nearest $\mathrm{mm}$.
Data Analysis: Spatial and temporal differences in salinity, temperature and dissolved oxygen, were subjected to different variance analysis of two ways variance analysis (ANOVA), normality and homogeneity standards of variances were double-checked by Kolgomorov-Smirnov and Bartlett tests, respectively. Density and biomass were determined as the number and weight of organisms per unit area (ind $\left./ \mathrm{m}^{2}, \mathrm{~g} / \mathrm{m}^{2}\right)$, extrapolated to $10 \mathrm{~m}^{2}$. Spatial and temporal differences in density and biomass were determined by two ways analysis of variance after transforming data to $\log (\mathrm{x}+1)$ and verifying homocedasticity. For this last analysis, extremely abundant species were eliminated (Clarke and Warwick 2001, Tsou and Matheson 2002). Community structure was analyzed through richness (S), evenness (J) and diversity of species (H') using Shannon-Wiener index. Dominance implying hierarchical structuring and evaluation of relative specie importance, was carried out in each zone and on annual basis with Index of Value of Importance (IVI), which incorporates information about density (DR), frequency (FR) and relative biomass (BR) for each species (IVI = DR + FR + BR) (Brower and Zar 1977).

Spatial and temporal changes in assemblage ecological parameters were performed with statistical program PRIMER 5, Plymouth Routines in Multivariate Ecological Research (Clarke and Gorley 2001). Fish assemblage differences among sites and/or climatic seasons were tested by Similarity analysis (ANOSIM) non-parametric test that applies the Bray Curtis similarity matrix (Clarke and Green 1988). Species contribution by climate season or sampling station was evaluated with similarity percentage (SIMPER) test, which determines percentage contribution of each species in order to classify a group (similarity) and discriminate species among sample groups (dissimilarity) (Clarke and Green 1988). Redundancy Analysis (RDA) was used to evaluate the interrelation among media densities and environmental parameters. This multi-varied analysis and IVI were performed with the statistical program ANACOM (De la Cruz 1994). Spearman 
correlation coefficient was used to determine the significance of each variable to fish distribution (Zar 1984). Species ecological classification was performed separating fish in marine-euryhaline, marine-stenohaline and estuarine residents species based on their relative abundance at each climate season (Castro Aguirre et al. 1999, Froese and Pauly 2004)

\section{RESULTS}

Hydrologic variables: Temperature was significantly different among all climatic seasons (rainy and norths $p=0.00014$; rainy and dry $p=0.002261$; norths and dry $p=0.00014$ ), with the lowest during norths $\left(24^{\circ} \mathrm{C}\right)$ and highest in the rainy $\left(31^{\circ} \mathrm{C}\right)$ (Fig. 1). Salinity showed the lowest values during norths (mainly in November) with an average of $29^{\circ} \mathrm{C}$, with the highest value $\left(37^{\circ} \mathrm{C}\right)$ in the dry season. No significant differences $(\mathrm{p}>0.05)$ were found for dissolved oxygen (Table 1).

Species composition and spatial-temporal variation: A total of 6474 fish were captured; comprising 81 species and 37 families, with a total weight of $64 \mathrm{~kg}$. Best represented families by number of species were Gerreidae (4 genus and 7 species), Carangidae (4 genus and 5 species), Sciaenidae (3 genus and 5 species), Engraulidae (1 genus and 5 species) and Syngnathidae (1 genus and 5 species). The most abundant species being Anchoa mitchilli, Fundulus persimilis, A. hepsetus, Eucinostomus gula, E. argenteus and Sphoeroides testudineus, while $S$. testudineus, F. persimilis and Mugil trichodon contributed with $56.6 \%$ of total weigh. Results of two-way ANOVA ( $>0.05)$ indicated not differences in density and biomass among climate seasons and sampling sites (Table 1). Considering both ecological parameters and their occurrence frequency 12 species contributed with more than $70 \%$, with S. testudineus (14.2) being the most representative (Fig. 2).

Diversity (H'), evenness and species richness did not vary significantly $(\mathrm{P}>0.05)$ among neither climate seasons nor sampling sites (Fig. 3). Spatially, average diversity was $2.40 \pm 0.69$ bits/ind, with maximum values recorded in site 5 (2.9 bits/ind) and minimum in site 1 (1.75 bits/ind). In the inlet of the lagoon (site 8) the
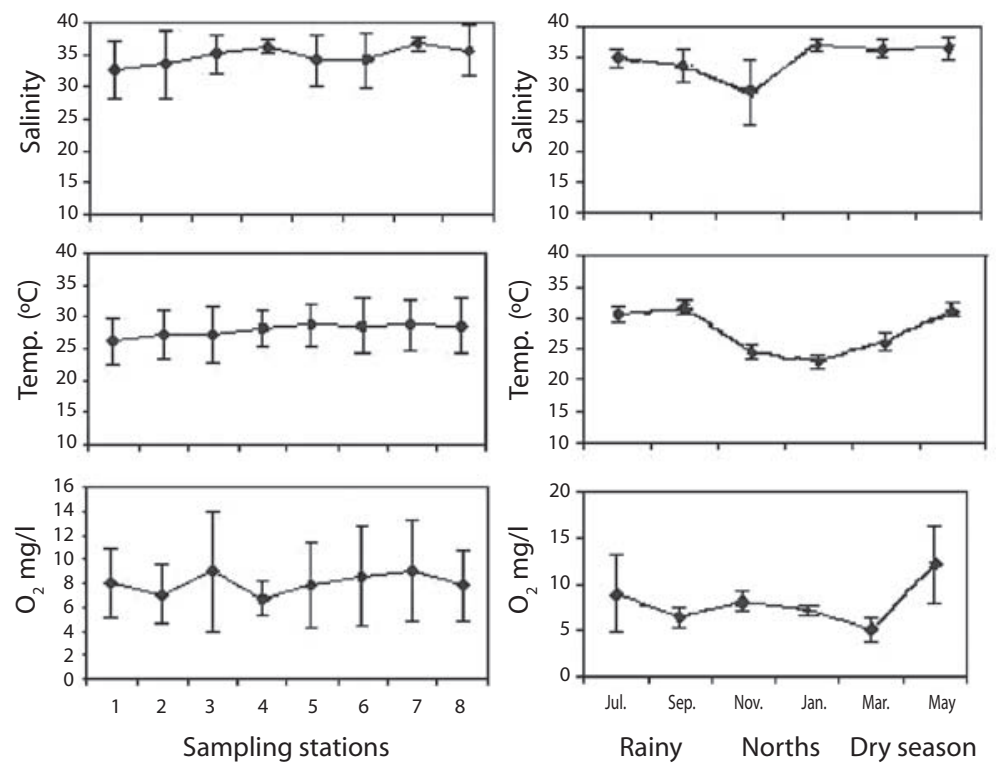

Fig. 1. Salinity, temperature and oxygen variations in sampling sites (A) and bimonthly (B) ( \pm 1 STD). 
highest species richness was recorded (17.67) and the lowest in site 2 (10.67). As to evenness, the highest corresponded to site $7(0.74)$ and the lowest in site 1 (0.52). On temporal basis, higher diversity was registered during norths (2.66 \pm 0.69 bits/ind), while the lowest was during dry season $(2.22 \pm 0.84$ bits/ind $)$. Likewise the highest species richness happened during norths (17.88) and the lowest during rains (10.63), and for evenness rainy season had the highest (0.68) and the lowest in dry season (0.62).

Ecological Category: Dominating fish with abundance higher than $70 \%$ in each of the climate seasons were classified as marine-euryhaline. Among these we found A. mitchilli, A. hepsetus, E. argenteus, E. gula, Lagodon rhomboides, Harengula jaguana, S. testudineus, and M. trichodon. Respect to marine-stenohaline component, higher percentage was shown in norths constituted by Opisthonema oglinum, Archosargus rhomboidalis, Haemulon bonariense, H. parrai, Anchoa cubana, A. lamprotaenia, Trachinotus falcatus and Sphyraena barracuda among others, while resident estuarine species were shown with higher incidence in dry season represented by $F$. persimilis, Floridichthys polyommus, Poecilia velifera, Gambusia yucatana,
TABLE 1

Results of ANOVA analysis to test significant differences in environmental variables (temperature, dissolved oxygen, salinity) and for some ecological parameters of fish community (diversity, species richness, evenness), density and biomass after their transformation $\log (x+1)$.

* Significant differences $p<0.05$

Variable/analysis result Spatial $(\mathrm{n}=8) \quad$ Temporal $(\mathrm{n}=3)$ Temperature

$\begin{array}{lll}F & 0.1800 & 81.0027\end{array}$

$\begin{array}{lll}p & 0.9857 & 0.0000^{*}\end{array}$

Dissolved oxygen

$\begin{array}{lll}F & 0.5549 & 0.6717\end{array}$

$\begin{array}{lll}p & 0.7810 & 0.5214\end{array}$

Salinity

$\begin{array}{lll}F & 0.8660 & 4.7104\end{array}$

$\begin{array}{lll}p & 0.5526 & 0.0204 *\end{array}$

Diversity Shannon $(\mathrm{H})$

$\begin{array}{lll}F & 0.6190 & 0.8737\end{array}$

$\begin{array}{lll}p & 0.7330 & 0.4320\end{array}$

No. species

$\begin{array}{lll}F & 0.4170 & 3.3861\end{array}$

$\begin{array}{lll}p & 0.8777 & 0.0531\end{array}$

Evenness (J)

$\begin{array}{lll}F & 0.7337 & 0.3603\end{array}$

$\begin{array}{lll}p & 0.6470 & 0.7016\end{array}$

Log Density

$\begin{array}{lll}F & 1.9433 & 0.5220 \\ p & 0.0750 & 0.5955\end{array}$

Log Biomass

$\begin{array}{lll}F & 2.1177 & 1.4126 \\ p & 0.0523 & 0.2501\end{array}$

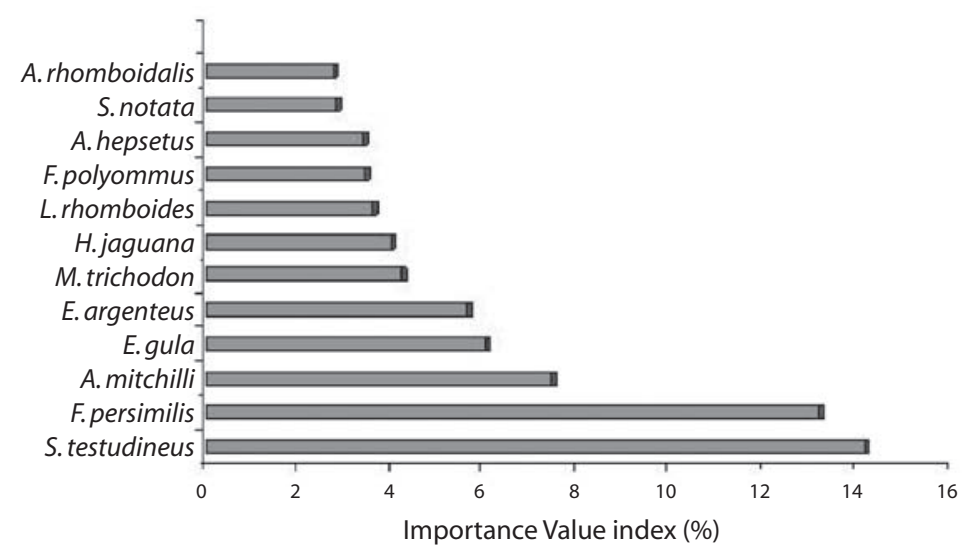

Fig. 2. Dominant fish species collected in "Bocas de Dzilam" lagoon during the study period, ranked by the Importance Value measure. 

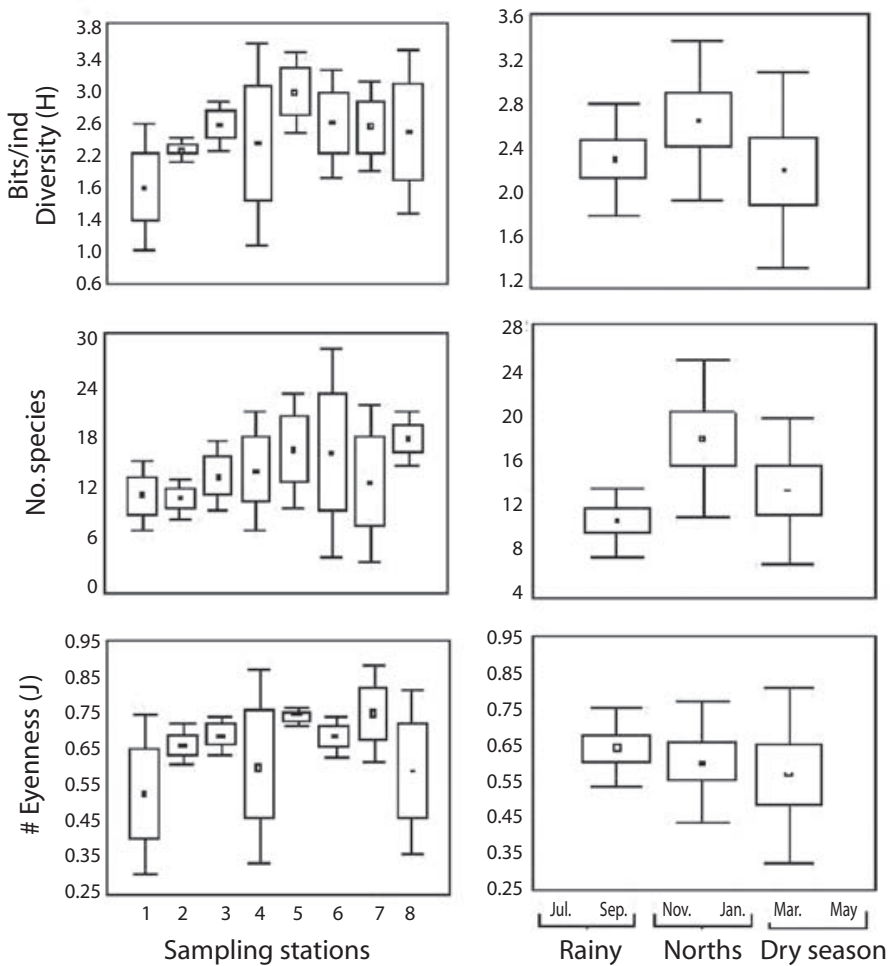

Fig. 3. Diversity index, total number of species and evenness of the sampling stations and season variation.

Cichlasoma urophthalmus, Lucania parva, Cyprinodon artifrons and Garmanella pulchra (Fig. 4, Table 2).

Spatial and temporal differences among assemblages: ANOSIM indicated significant differences in fish assemblage composition on the climate season $(\mathrm{R}=0.292, \mathrm{p}<0.05)$ and spatial $(\mathrm{R}=0.335, \mathrm{p}<0.001)$ among sites $1-4,1-6$, 1-7, 1-8, 5-7 and 6-8 (Table 3). Total significant dissimilarity among sampling sites shows a range between $83.09 \%$ and $93.62 \%$ (Table 4). These differences are caused by abundance among dominating species in each sampling site, being L. rhomboides, E. argenteus, E. gula, S. testudineus, H. jaguana, F. persimilis, F. polyommus, $A$. mitchilli, $M$. trichodon and $H$. parrai contributing with more than $50 \%$ to the spatial dissimilarity. Among climate seasons there is a total range of dissimilarity between
$78.62 \%$ and $83.99 \%$ with more than $50 \%$ by $E$. argenteus, E. gula, S. testudineus, F. polyommus, M. trichodon, L. rhomboides, H. jaguana, and F. persimilis (Table 4).

According to statistical test SIMPER, station 1 is characterized by $L$. rhomboides, which was found in $58.40 \%$ as well as other species such as $S$. testudineus, E. gula and E. argenteus which showed important percentages in the specific characterization of the sampling sites. There are stations where dominancy is shared and characterization is determined by many species that represent percentages lower than $30 \%$, such as sites 4,7 and 8 . Total similarity for each sampling site throughout time was from $5 \%$ (site 7 ) to $44 \%$ (site 2) showing the similarity degree of each site among different seasons. Temporally species common for rainy season were E. gula, E. argenteus, $S$. testudineus and $M$. trichodon with more than 


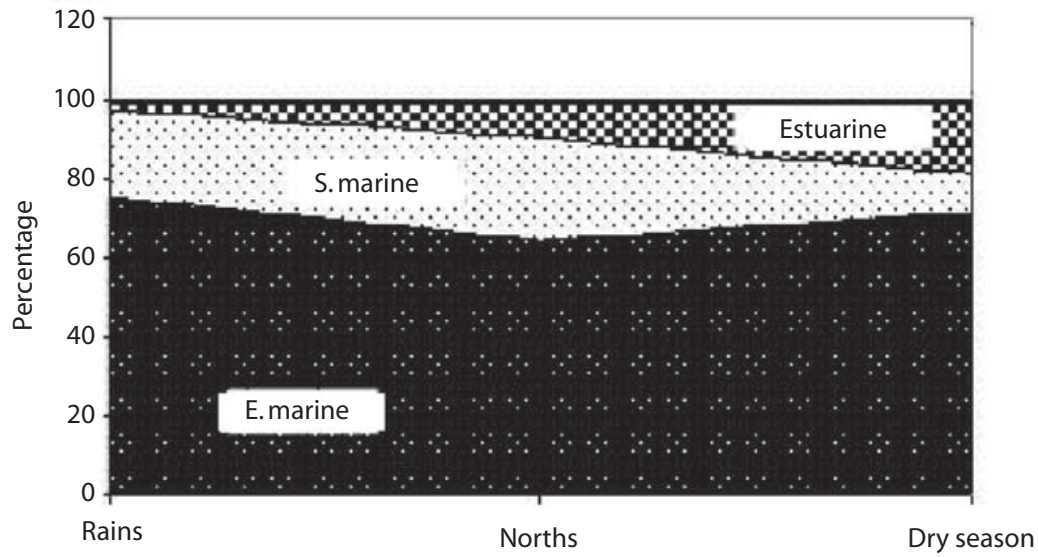

Fig. 4. Temporal distribution of ecological categories of the "Bocas de Dzilam" fish assemblages.

$60 \%$, furthermore norths are characterized by E. gula, E. argenteus, S. testudineus and F. polyommus, while dry season shows 8 species (E. argenteus, E. gula, S. testudineus, M. trichodon, L. rhomboides, H. jaguana, $H$. bonariense and $A$. lineatus) that contributed with more than $85 \%$.

Considering abundance and species composition, sampling sites are conformed by three main groups. The first one includes sites 7 and 8 , located in the external zone of the lagoon (marine zone) and characterized by the highest salinity value (35-38), species richness and evenness. In the second group, the site located in the inner zone (No.1) forms an independent entity with the lowest salinity record and the species richness and diversity because of the dominance of $L$. rhomboides. Finally, the third group is conformed by sites located in the center of the lagoon (2 - 6) (Fig. 5).

Relation between species and hydrologic variables: Species that showed a positive correlation (Spearman, $\mathrm{p}<0.05$ ) with salinity were Cynoscion arenarius, $A$. hepsetus, $T$. falcatus, Oligoplites saurus, $H$. jaguana, $O$. oglinum and Ariopsis felis, while Bairdiella chrysoura preferred oligohaline waters. In

TABLE 2

Relative abundance $(\% N)$ and biomass $(\% W)$, ecological category (E.C.) and seasonal occurrence (S.O.) of fish species registered in Dzilam lagoon, Yucatan

\begin{tabular}{|c|c|c|c|c|c|c|}
\hline Species & Family & $\% \mathrm{~N}$ & $\% \mathrm{~W}$ & E. C. & & S.O. \\
\hline Anchoa mitchilli & Engraulidae & 18.71 & 1.48 & E. mar & & N D \\
\hline Fundulus persimilis & Fundulidae & 18.18 & 20.77 & Estuar & & $\mathrm{D}$ \\
\hline Anchoa hepsetus & Engraulidae & 8.80 & 0.37 & E. mar & & $\mathrm{N}$ \\
\hline Eucinostomus gula & Gerreidae & 8.18 & 3.31 & E. mar & $\mathrm{R}$ & $\mathrm{N}$ \\
\hline Eucinostomus argenteus & Gerreidae & 8.11 & 2.54 & E. mar & $\mathrm{R}$ & $\mathrm{N}$ \\
\hline Sphoeroides testudineus & Tetraodontidae & 6.03 & 30.42 & E. mar & $\mathrm{R}$ & $\mathrm{N}$ \\
\hline Harengula jaguana & Clupeidae & 5.67 & 4.11 & E. mar & $\mathrm{R}$ & $\mathrm{N}$ \\
\hline Floridichthys polyommus & Cyprinodontidae & 4.73 & 1.28 & Estuar & $\mathrm{R}$ & $\mathrm{N}$ \\
\hline
\end{tabular}


TABLE 2 (Continued)

Relative abundance $(\% N)$ and biomass $(\% W)$, ecological category (E.C.) and seasonal occurrence (S.O.) of fish species registered in Dzilam lagoon, Yucatan

\begin{tabular}{|c|c|c|c|c|c|}
\hline Species & Family & $\% \mathrm{~N}$ & $\% \mathrm{~W}$ & E. C. & S.O. \\
\hline Lagodon rhomboides & Sparidae & 4.13 & 2.68 & E. mar & $\mathrm{R} N \mathrm{D}$ \\
\hline Mugil trichodon & Mugilidae & 3.02 & 5.39 & E. mar & $\mathrm{R} N \mathrm{D}$ \\
\hline Opisthonema oglinum & Clupeidae & 1.42 & 0.15 & S. mar & $\mathrm{N}$ \\
\hline Haemulon bonariense & Haemulidae & 1.36 & 2.43 & S. mar & $\mathrm{R} N \mathrm{D}$ \\
\hline Archosargus rhomboidalis & Sparidae & 1.28 & 2.68 & S. mar & $\mathrm{R} N \mathrm{D}$ \\
\hline Poecilia velifera & Poeciliidae & 1.16 & 0.09 & Estuar & $\mathrm{N} D$ \\
\hline Strongylura notata & Belonidae & 1.05 & 4.87 & E. mar & $\mathrm{R} N \mathrm{D}$ \\
\hline Selene vomer & Carangidae & 0.71 & 1.84 & E. mar & $\mathrm{N} D$ \\
\hline Achirus lineatus & Achiridae & 0.61 & 0.19 & E. mar & $\mathrm{R} N \mathrm{~N}$ \\
\hline Haemulon parra & Haemulidae & 0.60 & 0.43 & S. mar & $\mathrm{R} \mathrm{N}$ \\
\hline Sphoeroides nephelus & Tetraodontidae & 0.43 & 0.43 & E. mar & $\mathrm{N} D$ \\
\hline Anchoa cubana & Engraulidae & 0.43 & 0.02 & S. mar & $\mathrm{N}$ \\
\hline Lutjanus griseus & Lutjanidae & 0.39 & 0.71 & E. mar & $\mathrm{R} N \mathrm{D}$ \\
\hline Ariopsis felis & Ariidae & 0.39 & 2.53 & E. mar & $\mathrm{R} N \mathrm{~N}$ \\
\hline Anchoa lamprotaenia & Engraulidae & 0.38 & 0.17 & S. mar & $\mathrm{N} D$ \\
\hline Mugil curema & Mugilidae & 0.37 & 0.21 & E. mar & $\mathrm{R} N \mathrm{D}$ \\
\hline Gambusia yucatana & Poeciliidae & 0.30 & 0.007 & Estuar & $\mathrm{N} D$ \\
\hline Opsanus beta & Batrachoididae & 0.26 & 0.24 & E. mar & $\mathrm{N} D$ \\
\hline Orthopristis chrysoptera & Haemulidae & 0.25 & 0.91 & E. mar & $\mathrm{R} \quad \mathrm{N} \quad \mathrm{D}$ \\
\hline Strongylura timucu & Belonidae & 0.21 & 0.87 & E. mar & $\mathrm{Ll} \mathrm{D}$ \\
\hline Synodus foetens & Synodontidae & 0.21 & 0.37 & E. mar & $\mathrm{R} \quad \mathrm{N} \mathrm{D}$ \\
\hline Trachinotus falcatus & Carangidae & 0.20 & 1.10 & S. mar & $\mathrm{R} N \mathrm{D}$ \\
\hline "Cichlasoma" urophthalmus & Cichlidae & 0.20 & 1.79 & Estuar & $\mathrm{N} D$ \\
\hline Elops saurus & Elopidae & 0.17 & 0.55 & E. mar & $\mathrm{R} \mathrm{N}$ \\
\hline Oligoplites saurus & Carangidae & 0.10 & 0.08 & E. mar & $\mathrm{R} \mathrm{N}$ \\
\hline Cynoscion arenarius & Sciaenidae & 0.08 & 0.04 & E. mar & $\mathrm{N}$ \\
\hline Cynoscion nebulosus & Sciaenidae & 0.08 & 0.21 & E. mar & $\mathrm{R} \quad \mathrm{N} \quad \mathrm{D}$ \\
\hline Lucania parva & Fundulidae & 0.08 & 0.001 & Estuar & $\mathrm{N} D$ \\
\hline Paralichthys albigutta & Paralichthyidae & 0.08 & 0.03 & E. mar & $\mathrm{R} \mathrm{D}$ \\
\hline Scorpaena brasiliensis & Scorpaenidae & 0.07 & 0.02 & E. mar & $\mathrm{N}$ \\
\hline Prionotus tribulus & Triglidae & 0.07 & 0.05 & E. mar & $\mathrm{N} D$ \\
\hline Sphyraena barracuda & Sphyraenidae & 0.07 & 1.39 & S. mar & $\mathrm{R} N \mathrm{~N}$ \\
\hline Menticirrhus saxatilis & Sciaenidae & 0.07 & 0.07 & E. mar & $\mathrm{N} D$ \\
\hline Hyporhamphus roberti & Hemiramphidae & 0.06 & 0.01 & E. mar & $\mathrm{N}$ \\
\hline Bairdiella chrysoura & Sciaenidae & 0.06 & 0.26 & E. mar & $\mathrm{D}$ \\
\hline Stephanolepis hispidus & Monacanthidae & 0.05 & 0.007 & S. mar & $\mathrm{N}$ \\
\hline Chilomycterus schoepfii & Diodontidae & 0.05 & 0.009 & E. mar & $\mathrm{N}$ \\
\hline Symphurus plagiusa & Cynoglossidae & 0.05 & 0.02 & E. mar & $\mathrm{N} D$ \\
\hline
\end{tabular}


TABLE 2 (Continued)

Relative abundance $(\% N)$ and biomass $(\% W)$, ecological category (E.C.) and seasonal occurrence (S.O.) of fish species registered in Dzilam lagoon, Yucatan

\begin{tabular}{|c|c|c|c|c|c|}
\hline Species & Family & $\% \mathrm{~N}$ & $\% \mathrm{~W}$ & E. C. & S.O. \\
\hline Cyprinodon artifrons & Cyprinodontidae & 0.05 & 0.001 & Estuar & $\mathrm{D}$ \\
\hline Chriodorus atherinoides & Hemiramphidae & 0.05 & 0.09 & E. mar & $\mathrm{D}$ \\
\hline Menidia colei & Atherinopsidae & 0.05 & 0.002 & E. mar & $\mathrm{N} D$ \\
\hline Nicholsina usta & Scaridae & 0.03 & 0.01 & S. mar & $\mathrm{N}$ \\
\hline Gerres cinereus & Gerreidae & 0.03 & 0.02 & E. mar & $\mathrm{N}$ \\
\hline Haemulon plumierii & Haemulidae & 0.03 & 0.02 & S. mar & $\mathrm{N}$ \\
\hline Chaetodipterus faber & Ephippidae & 0.03 & 0.02 & E. mar & $\mathrm{N} D$ \\
\hline Syngnathus louisianae & Syngnathidae & 0.03 & 0.002 & E. mar & $\mathrm{N} \quad \mathrm{D}$ \\
\hline Dasyatis americana & Dasyatidae & 0.02 & 1.39 & E. mar & $\mathrm{R}$ \\
\hline Anchoa lyolepis & Engraulidae & 0.02 & 0.002 & E. mar & $\mathrm{N}$ \\
\hline Citharichtys spilopterus & Paralichtyidae & 0.02 & 0.008 & E. mar & $\mathrm{D}$ \\
\hline Eucinostomus melanopterus & Gerreidae & 0.02 & 0.001 & E. mar & $\mathrm{N}$ \\
\hline Syngnathus floridae & Syngnathidae & 0.02 & 0.002 & E. mar & $\mathrm{N}$ \\
\hline Lutjanus cyanopterus & Lutjanidae & 0.01 & 0.01 & E. mar & $\mathrm{R}$ \\
\hline Hyporhamphus meeki & Hemirramphidae & 0.01 & 0.01 & E. mar & $\mathrm{D}$ \\
\hline Acanthostracion quadricornis & Ostraciidae & 0.01 & 0.23 & E. mar & $\mathrm{R}$ \\
\hline Diapterus auratus & Gerreidae & 0.01 & 0.01 & E. mar & $\mathrm{N}$ \\
\hline Eugerres plumieri & Gerreidae & 0.01 & 0.07 & E. mar & $\mathrm{D}$ \\
\hline Gymnura micrura & Gymnuridae & 0.01 & 0.08 & S. mar & $\mathrm{R}$ \\
\hline Bathygobius soporator & Gobiidae & 0.01 & 0.002 & E. mar & $\mathrm{R}$ \\
\hline Ctenogobius stigmaticus & Gobiidae & 0.01 & 0.001 & E. mar & $\mathrm{D}$ \\
\hline Garmanella pulchra & Cyprinodontidae & 0.01 & $<0.001$ & Estuar & $\mathrm{D}$ \\
\hline Opsanus phobetron & Batrachoididae & 0.01 & 0.12 & S. mar & $\mathrm{N}$ \\
\hline Bairdiella sanctaeluciae & Sciaenidae & 0.01 & 0.01 & S. mar & $\mathrm{N}$ \\
\hline Syngnathus scovelli & Syngnathidae & 0.01 & $<0.001$ & E. mar & $\mathrm{D}$ \\
\hline Carangoides ruber & Carangidae & 0.01 & 0.007 & E. mar & $\mathrm{R}$ \\
\hline Monacanthus ciliatus & Monacanthidae & 0.01 & 0.001 & S. mar & $\mathrm{N}$ \\
\hline Caranx latus & Carangidae & 0.01 & 0.001 & E. mar & $\mathrm{N}$ \\
\hline Archosargus probatocephalus & Sparidae & 0.01 & 0.004 & E. mar & $\mathrm{R}$ \\
\hline Diapterus rhombeus & Gerreidae & 0.01 & 0.001 & E. mar & $\mathrm{N}$ \\
\hline Syngnathus pelagicus & Syngnathidae & 0.01 & 0.001 & E. mar & $\mathrm{D}$ \\
\hline Mugil cephalus & Mugilidae & 0.01 & 0.03 & E. mar & $\mathrm{R}$ \\
\hline Pomatomus saltatrix & Pomatomidae & 0.01 & 0.01 & S. mar & $\mathrm{N}$ \\
\hline Urobatis jamaicensis & Urolophidae & 0.01 & 0.39 & E. mar & $\mathrm{N}$ \\
\hline Syngnathus makaxi & Syngnathidae & 0.01 & $<0.001$ & E. mar & $\mathrm{D}$ \\
\hline
\end{tabular}


TABLE 3

$R$ statistic values and their significance levels for pairwise comparisons of species composition of the sites and seasons using ANOSIM $(P<0.05)$

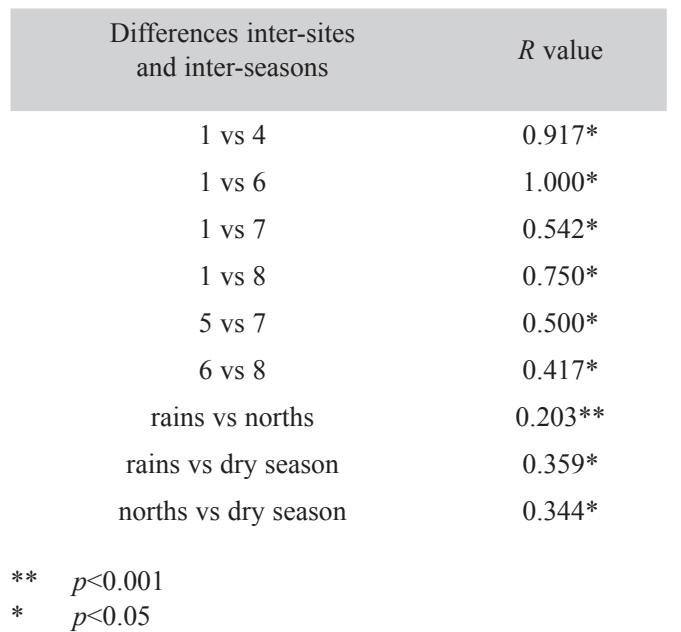

terms of dissolved oxygen Achirus lineatus and Dasyatis americana showed a significant negative relation (Spearman, $\mathrm{p}>0.05$ ). Temperature affected significantly Symphurus plagiusa, B. chrysoura and Sphoeroides nephelus, while A. lamprotaenia, M. trichodon and $A$. felis were found in areas with higher temperature values (Table 5).

RDA analysis indicated that salinity and temperature were the principal hydrologic factors that influence composition and species distribution. The first two axes explain 41.14\% of variance and correlation (0.93 and 0.56) with $A$. mitchilli and $A$. hepsetus occurrence greatly influenced by salinity and temperature (Fig. 6). Some species distribution such as $L$. rhomboides, F. persimilis, $H$. jaguana, and $S$. testudineus were influenced by other variables not measured in this research, such as substrate type, surrounding vegetation, food availability and even specific biological considerations.
TABLE 4

Species contributing to the dissimilarities between sampling stations and climatic seasons based on relative abundance

\begin{tabular}{|c|c|c|c|}
\hline $\begin{array}{l}\text { Groups } \\
\text { compared }\end{array}$ & Dissimilarity & Species & $\%$ \\
\hline $\mathrm{S} 1-\mathrm{S} 4$ & 87.77 & $\begin{array}{l}\text { L. rhomboides } \\
\text { F. persimilis } \\
\text { H. jaguana } \\
\text { E. argenteus }\end{array}$ & $\begin{array}{c}18.95 \\
17.24 \\
10.08 \\
9.83\end{array}$ \\
\hline S1-S6 & 86.03 & $\begin{array}{l}\text { L. rhomboides } \\
\text { E. argenteus } \\
\quad \text { E. gula }\end{array}$ & $\begin{array}{c}24.63 \\
18.56 \\
9.80\end{array}$ \\
\hline S1-S7 & 93.62 & $\begin{array}{l}\text { L. rhomboides } \\
\text { E. gula } \\
\text { S. testudineus } \\
\text { A. rhomboidalis }\end{array}$ & $\begin{array}{l}29.95 \\
11.06 \\
8.15 \\
6.97\end{array}$ \\
\hline S1-S8 & 90.64 & $\begin{array}{l}\text { L. rhomboides } \\
\quad \text { E. gula } \\
\text { A. mitchilli } \\
\text { M. trichodon } \\
\text { H. parrai }\end{array}$ & $\begin{array}{l}19.89 \\
9.11 \\
8.75 \\
8.10 \\
6.21\end{array}$ \\
\hline S5-S7 & 88.14 & $\begin{array}{l}\text { E. argenteus } \\
\text { E. gula } \\
\text { F. polyommus } \\
\text { S. testudineus }\end{array}$ & $\begin{array}{l}15.39 \\
14.41 \\
11.55 \\
10.15\end{array}$ \\
\hline S6-S8 & 83.09 & $\begin{array}{c}\text { E. argenteus } \\
\text { E. gula } \\
\text { A. mitchilli } \\
\text { M. trichodon } \\
\text { H. parrai } \\
\text { F. polyommus }\end{array}$ & $\begin{array}{l}15.99 \\
10.03 \\
8.80 \\
7.63 \\
6.16 \\
6.16\end{array}$ \\
\hline $\begin{array}{l}\text { Rains- } \\
\text { norths }\end{array}$ & 78.62 & $\begin{array}{l}\text { E. argenteus } \\
\quad \text { E. gula } \\
\text { S. testudineus } \\
\text { F. polyommus } \\
\text { M. trichodon }\end{array}$ & $\begin{array}{l}13.64 \\
11.90 \\
9.67 \\
8.90 \\
8.24\end{array}$ \\
\hline $\begin{array}{l}\text { Rains- } \\
\text { dry } \\
\text { season }\end{array}$ & 80.91 & $\begin{array}{l}\text { E. argenteus } \\
\text { E. gula } \\
\text { L. rhomboides } \\
\text { M. trichodon }\end{array}$ & $\begin{array}{c}14.61 \\
14.55 \\
11.60 \\
9.93\end{array}$ \\
\hline $\begin{array}{l}\text { Norths } \\
\text {-dry } \\
\text { season }\end{array}$ & 83.99 & $\begin{array}{l}\text { S. testudineus } \\
\text { E. gula } \\
\text { F. polyommus } \\
\text { E. argenteus } \\
\text { L. rhomboides } \\
\text { H. jaguana } \\
\text { F. persimilis }\end{array}$ & $\begin{array}{l}10.95 \\
8.59 \\
8.06 \\
8.04 \\
6.20 \\
5.94 \\
5.91\end{array}$ \\
\hline
\end{tabular}


TABLE 5

Correlation matrix of overall means of hydrologic variables with fish species (only those species with at least one significant correlation are shown); $*_{p}<0.05$

\begin{tabular}{lccc}
\multicolumn{1}{c}{ Species } & Salinity & Oxygen & Temperature \\
Symphurus plagiusa & -1.344 & -1.458 & $-2.954^{*}$ \\
Cynoscion arenarius & $2.898^{*}$ & 0.096 & 0.759 \\
Bairdiella chrysoura & $-2.636^{*}$ & -0.764 & $-2.636^{*}$ \\
Sphoeroides nephelus & -2.214 & -1.199 & $-2.445^{*}$ \\
Achirus lineatus & -0.608 & $-2.690^{*}$ & 0.388 \\
Anchoa lamprotaenia & 1.837 & 0.515 & $3.167^{*}$ \\
Anchoa hepsetus & $3.167 *$ & -0.408 & 1.188 \\
Mugil trichodon & 1.084 & 0.572 & $2.500^{*}$ \\
Trachinotus falcatus & $3.167 *$ & -0.408 & 1.188 \\
Oligoplites saurus & $3.088^{*}$ & -0.648 & 1.064 \\
Harengula jaguana & $3.880^{*}$ & -0.699 & 0.848 \\
Opisthonema oglinum & $4.381^{*}$ & -0.236 & 1.098 \\
Ariopsis felis & $2.705^{*}$ & 0.000 & $3.613^{*}$ \\
Dasyatis americana & 0.191 & $-2.940 *$ & -0.894
\end{tabular}

\section{DISCUSSION}

Species composition and ecological category: Compared with previous studies and with other coastal systems in Yucatan Peninsula and Gulf of Mexico, fish species richness of "Bocas de Dzilam" (81 spp.) can be considered high (Vega- Cendejas et al. 1997, Raz-Guzmán and Huidobro 2002, Vega-Cendejas and Hernández 2004) (Table 6). However, many of the species are represented by low densities because they are in transit and enter to the system for very short terms, usually only to feed themselves (Strongylura notata) or sometimes to lay eggs ( $F$. persimilis). According to Simier et al. (2004) the high diversity registered can be caused by: 1) establishment in the estuary of many marine species due to the permanent communication with the ocean and the presence of various freshwater seeps; and 2) heterogeneous habitats (seagrass meadows, prop root mangroves, mudflats, underground water flowerings) favoring colonization by different fish species.

The ichthyofauna in "Bocas de Dzilam" is dominated by estuarine species, characteristic

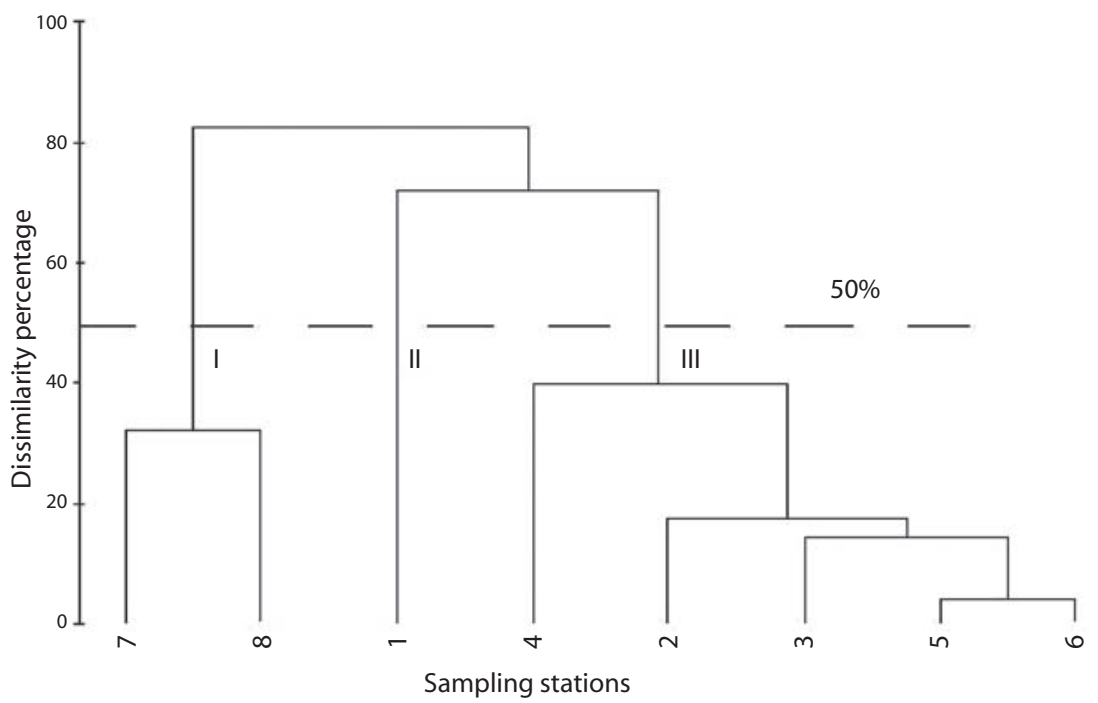

Fig. 5. Dendrogram of dissimilarity of stations based on $\log (x+1)$ transformed total abundance of fish species using the Bray Curtis index. 


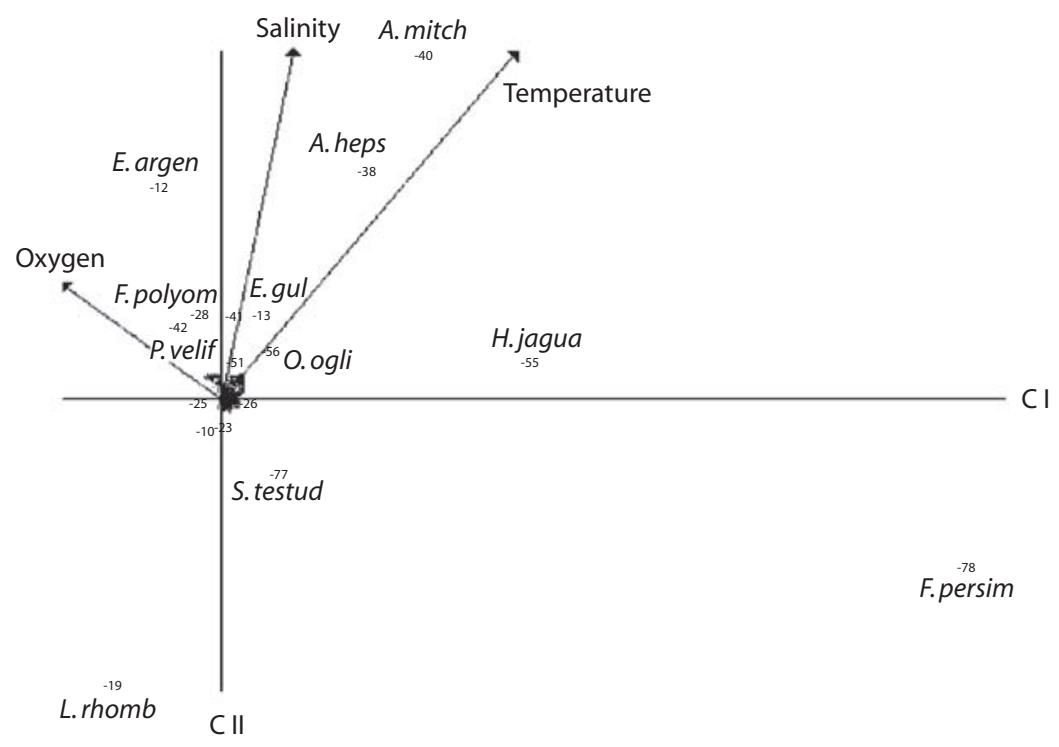

Fig. 6. Redundancy analyses (RDA) diagram of the overall fish abundances versus environmental variables represented by arrows. Species abbreviations are the first letter of the genus name and first four letters of the species name.

TABLE 6

Comparative fish species number and coastal lagoon total area for some systems from Yucatan Peninsula and Gulf of Mexico

$\begin{array}{lccl}\text { Lagoon } & \text { No Spp } & \text { Area } & \text { Authors } \\ \text { Bocas de Dzilam } & 81 & 9.4 \mathrm{~km}^{2} & \text { Present study } \\ \text { Rio Lagartos } & 81 & 80 \mathrm{~km}^{2} & \text { Vega-Cendejas and Hernández, } 2004 \\ \text { Celestún lagoon } & 94 & 28.14 \mathrm{~km}^{2} & \text { Vega-Cendejas, 2004 } \\ \text { Laguna Madre } & 84 & 200 \mathrm{~km}^{2} & \text { Raz-Guzmán and Huidobro, } 2002\end{array}$

of other coastal lagoons of the Yucatan Peninsula, such as mojarras and anchovies (Vega Cendejas et al. 1997, Vega Cendejas and Hernández 2004). Moreover, many commercial importance species (Haemulon plumierii, Orthopristis chrysoptera, Cynoscion nebulosus, C. arenarius, Lutjanus griseus, A. mitchilli, $A$. hepsetus, E. gula and E. argenteus,) used the system for feeding, raising and shelter. Tough salinity gradients create physiological barriers for most species, it has been found that seasonally, the marine euryhaline component, like $S$. notata enter to these coastal systems taking advantage of the diverse food resources Many others use this environment as nursery such as L. griseus, E. argenteus and E. gula. The small size recorded for most of the individuals indicates the importance of the seagrass meadows, mangroves and mudflat to grow and shelter against predators. Similar results were reported by Vega-Cendejas and Hernández (2004) for Río Lagartos lagoon.

Temporal and spatial variations among assemblages: Temporally, during norths the highest species number (59) uses the lagoon as 
protection against strong winds and was characterized by their abundance and aggregation, such as anchovies (A. mitchilli and A. hepsetus) and jacks (Selene vomer). High density and biomass percentages are generally associated to the occasional presence of small pelagic fish big schools (Simier et al. 2004). Seasonal variability in fish assemblages reflects some species abundance due to reproductive pulses, mainly during the first stages in their life cycle (Machado and Araújo 2003). For instance, during the dry season, it was recorded a $F$. persimilis abundance peak in relation to its reproductive stage. Similar results were found by Vega-Cendejas and Hernández (2004) that reported an abundance peak for $F$. grandissimus during dry season in Río Lagartos, suggesting a reproductive season for this species in the Yucatan coast.

In terms of spatial distribution, the inner zone (station 1), showed the least number of species (20), and Lagodon rhomboides was the most abundant with more than $60 \%$ of the total capture. On the other hand, stations with the highest number of species were 6 and 8 . In site 6 , located in the innermost zone of the lagoon, many estuarine-type and freshwater species were found (11\%) such as "Cichlasoma" urophtalmus, C. artifrons, G. pulchra, G. yucatana, P. velifera and Mendia colei. This site is characterized by freshwater seeps that give hydrologic estuarine conditions and produce an abundance of organic matter (personal observation), which may explain the presence of these species even if salinity is high; furthermore this site is characterized by a high habitat heterogeneity with mangroves and muddy flatland floors that contribute to ichthyofauna diversification, even for shelter of important species such as " $C$ ". urophtalmus; moreover they provide food to benthophagous (Gerreidae) and detritophagous species (Mugilidae) (Simier et al. 2004). Station 8, located in the external zone (near the inlet), is the other site with the highest species richness in the lagoon. This place has a permanent communication with the sea, favoring transit for many interacting species. Predominantly open estuaries are species rich; their permanent or near-permanent connection with the sea allows access into these systems (Harrison and Whitfield 2006).

According to abundance and species composition, sampling sites were distributed in three groups (Figure 5), the first one included stations located in the external zone (7 and 8), where specific composition comprises mainly marine species considered only in transit and that occasionally get into the lagoon. The use of surf zones by a great number of fish, mainly in juvenile stages, is likely responsible for the existence of rich sources of food in form of zooplankton and for the protection against predators that those shallow, low turbid and low turbulent waters provide (Lasiak 1986).

The second group is conformed by site 1 located in the inner zone, which forms an independent group due to a high representation of small size individuals $(3-5 \mathrm{~cm})$ of $L$. rhomboides. This zone is characterized by a bed of submerged vegetation dominated by Halodule wrigthii (Herrera Silveira et al. 1998). This shows the ecological role of seagrass meadows as growing and feeding zones for juvenile stages of marine species (Jenkins et al. 1997, Gray et al. 1998). The third group comprises inner stations, with dominating species of wide distribution within the lagoon. These sites are also characterized by shallow depths with muddy bottoms, sometimes with submerged macrophytes and macroalgae, aside from high turbidness and a great quantity of decomposing organic matter, which is used by detritophagous species.

Relation between species and hydrologic variables: The ever-changing conditions in hydrology can become a physical barrier for the access of many marine origin species; such is the case of salinity gradients, while variations in temperature can affect ichthyofauna distribution, through their thermal differences, but above all because of the joined effect with dissolved oxygen (Marshall and Elliott 1998). Environmental variables did not show spatial gradient as in other coastal systems of Yucatan 
Peninsula (Rueda 2001, Vega-Cendejas and Hernández 2004). However there is a clear seasonal pattern influenced by climatic conditions, specially during norths. Species found as estuarine residents and most dominating did not show any relation with environmental variables, but some marine species did so because of their abundance in certain climatic seasons. Salinity has a great influence over distribution and abundance of some marine species, like in anchovies ( $A$. hepsetus and A. mitchilli) and herrings (H. jaguana and $O$. oglinum). We have to take into account that response of many species to salinity fluctuations can vary during their life cycle. For instance, juvenile herrings can tolerate wider ranges than adults (Marshall and Elliott 1998). As for temperature, it showed certain influence over some species, such as mullets (M. trichodon), silver croaker (B. chrysoura) and catfish (A. felis). It is unlikely that thermal tolerance by itself be the main factor to influence the examined species distribution. However, temperature's influence over fish distribution grows through effects of synergy between temperature and dissolved oxygen (Pomfret et al. 1991, Blaber 1997, Marshall and Elliott 1998). We have to consider that biological interactions that influence the composition of estuarine fish assemblages, such as, habitat preference, predator-prey relation, food availability and often reproductive biology of species. Therefore, estuarine fish patterns of distribution can not be only attributed to physicochemical factors, but to a synergy between biotic and abiotic factors.

\section{CONCLUSIONS}

The main spatial and temporal differences between fish assemblages in a coastal system influenced by freshwater seeps was species composition, due to the behavior and biology of each species at a given time and place. Site 1, located in the inner zone (bottom with Halodule wrightii) showed the greatest difference amongst all sampling sites. In addition it can be characterized as having a lower diversity value because of juvenile dominance
(L. rhomboides) i.e. using the system as a nursery. Temporarily, the greatest diversity and species richness was obtained during the north wind season because several marine species of ecological and economical importance used the system for shelter and feeding. Salinity was the hydrologic variable that best related fish distribution patterns with a direct influence on the pelagic species $A$. mitchilli, $A$. hepsetus, $H$. jaguana and O. oglinum.

\section{ACKNOWLEDGMENTS}

We are grateful for the help of many colleagues that was indispensable to realize the fieldworks. In special we want to thank Mirella Hernández de Santillana for her assistance at fish sampling and in the laboratory- thank you to all of you. We are indebted to Luis Capurro for corrections to the English versions and to anonymous referees for their comments and suggestions that enrich the manuscript.

\section{RESUMEN}

Las lagunas costeras son sistemas importantes para muchas especies de organismos dulceacuícolas, estuarinos y marinos, ya que son consideradas zonas de reproducción, refugio y alimentación de muchas especies de peces. El presente estudio analizó los ensamblajes de la comunidad íctica de la reserva de Dzilam y su relación con las variables hidrológicas. Se capturaron un total de 6474 individuos (81 especies), en donde Sphoeroides testudineus, Fundulus persimilis, Anchoa mitchilli, Eucinostomus gula, Eucinostomus argenteus and Mugil trichodon contribuyeron con más del 50\% del Indice de Valor de Importancia (IVI). Las diferencias en composición de especies se encontraron entre temporadas, la mayor durante los nortes. Espacialmente, las diferencias fueron relacionadas con la presencia de afloramientos de agua dulce, la mayor en la zona Este de la laguna y la menor con mayor diferencia en composición específica se localizó en la parte Oeste de la zona interna, esto debido a la gran abundancia y dominancia de una sola especie (L. rhomboides). La salinidad y la temperatura fueron las variables que presentaron la mayor influencia en la distribución de algunas especies pelágicas como son $A$. mitchilli y $A$. hepsetus. Debido a la abundancia de afloramientos de agua dulce, característicos de las lagunas costeras de la Península de Yucatán, los ensamblajes de peces muestran diferencias espaciales y temporales en la composición específica. 
Palabras clave: Ictiofauna, composición de especies, variación temporal, entradas de agua dulce, variables ambientales, Bocas de Dzilam.

\section{REFERENCES}

Blaber S., J.M. 1997. Fish and fisheries of tropical estuaries. Chapman and Hall, London, England.

Blaber S., J.M. \& T.G. Blaber. 1980. Factors affecting the distribution of juvenile estuarine and inshore fish. J. Fish Biol. 17: 143-162.

Brower JE, Zar JH. 1977. Field and Laboratory Methods for General Ecology. W. C. Brown Co. Publishers, Dubuque, Iowa, USA.

Castro-Aguirre JP, Espinoza H, Schmitter-Soto J. 1999. Ictiofauna estuarino lagunar y vicaria de México. Colección Textos Politecnicos. Serie Biotecnologias. Ed. Limusa, México.

Clarke K.R. \& R.H.Green 1988. Statistical design and analysis for a 'biological effects' study. Mar. Ecol. Prog. Series. 46: 213-226.

Clarke KR, Gorley R. 2001. PRIMER V5: User Manual/ Tutorial. PRIMER-E Plymouth, UK.

Clarke KR, Warwick RM. 2001. Change in Marine Communities: An Approach to Statistical Analysis and Interpretation. $2^{\text {nd }}$ edition. Natural Environment Research Council, Plymouth Marine Laboratory, Plymouth U.K.

De La Cruz AG. 1994. ANACOM: Sistema para el Análisis de Comunidades en computadoras personales. Versión 3.0. Manual del usuario. CINVESTAVIPN, Unidad Mérida, Yucatán, México.

Francis M., R. Hurst, B. McArdle, N. Bagley \& O. Anderson. 2002. New Zeland demersal fish assemblages. Env. Biol. Fish. 65: 215-234.

Gray C. A., R.C. Chick \& D.J. McElligott. 1998. Diel changes in assemblages of fishes associated with shallow seagrass and bare sand. Estuar. Coast. Shelf Sci. 46: 849-859.

Harrison, D.T. \& A.K. Whitfield. 2006. Estuarine typology and the structuring of fish communities in South Africa. Env. Biol. Fish. 75: 269-293.

Herrera-Silveira J.A., R.J. Ramírez \& A. Zaldivar. 1998. Overview and characterization of the hydrology and primary producer communities of selected coastal lagoons of Yucatan, México. Aquatic Ecosystem Health and Management. 1: 353-372.
Jenkins GP, May HMA, Wheatley MJ, Holloway MG. 1997. Comparison of fish assemblages associated with seagrass and adjacent unvegetated habitats of Port Phillip Bay and Corner Inlet, Victoria, Australia, with emphasis on commercial species. Estuar. Coast. Shelf Sci. 44: 569-588.

Kupschus S \& D. Tremain. 2001. Associations between fish assemblages and environmental factors in nearshore habitats of a subtropical estuary. J. Fish Biol. 58: 1383-1403.

Lasiak TA. 1986. Juveniles, food and the surfzone habitat: implications for teleost nursery areas. South African J. Zoo. 21: 52-56.

Machado P.A.L. \& F.G. Araújo. 2003. Spatial, temporal, and diel variations of fish assemblages at two sandy beaches in the Sepetiba Bay, Rio de Janeiro, Brazil. Estuar. Coast. Shelf Sci. 57: 817-828.

Marchand J. 1993. The influence of seasonal salinity and turbidity maximum variations on the nursery function of the Loire estuary (France). Netherlands J. Aquat. Ecol. 27: 427-436.

Marshall S. \& M. Elliot. 1998. Environmental Influences on the Fish Assemblage of the Humber Estuary, U.K. Estuar. Coast. Shelf Sci. 46: 175-184.

Medina-Gómez I, Herrera-Silveira J.A. 2003. Spatial characterization of water quality in a karstic coastal lagoon without anthropogenic disturbance: a multivariate approach. Estuar. Coast. Shelf Sci. 58: 455-465.

Pomfret JR, Elliott M, O`Reilly MG \& Phillips S. 1991. Spatial and temporal patterns in the fish communities in two U.K. North Sea estuaries, p.277-284. In: Elliott M \& Ducrotoy SP (eds) Estuaries and coasts: Spatial and Temporal Intercomparisons. Olsen \& Olsen, Fredensborg, Denmark.

Raz-Guzman A \& L. Huidobro. 2002. Fish communities in two environmentally different estuarine systems of Mexico. J. Fish Biol. 61: 182-195.

Rueda M. 2001. Spatial distribution of fish species in a tropical estuarine lagoon: a geostatistical appraisal. Mar. Ecol. Prog. Ser. 222: 217-226.

Simier M, L. Blanc, C. Aliaume, P.S. Diouf \& J.J. Albaret. 2004. Spatial and temporal structure of fish assemblages in an "inverse estuary", the Sine Saloum system (Senegal). Estuar. Coast. Shelf Sci. 59: 69-86.

Tsou TS, Matheson RE Jr. 2002. Seasonal Changes in the Nekton Community of the Suwanee River Estuary and the Potential Impacts of Freshwater Withdrawal. Estuaries 25: 1372-1381. 
Vega-Cendejas ME. 2004. Ictiofauna de la Reserva de la Biosfera Celestún, Yucatán: una contribución al conocimiento de su diversidad. An. Inst. Biol. Univ. Nal. Autón. Méx. Ser. Zoo. 75: 193-2006.

Vega-Cendejas M.E. \& M. Hernández. 2004. Fish community structure and dynamics in a coastal hypersaline lagoon: Rio Lagartos, Yucatan, Mexico. Estuar. Coast. Shelf Sci. 60: 285-299.

Vega-Cendejas ME, F, Arreguin \& M. Hernández. 1993 Trophic fluxes on the campeche, Campeche Bank, México. pp. 206-213 In Christensen V. and Pauly D. (eds) Trophic models of aquatic ecosystems, ICLAEM Conf. Proc.

Vega-Cendejas M.E., M. Hernández \& F. Arreguin. 1994.

Trophic interrelations in a beach seine fishery from the northwestern coast of the Yucatan peninsula, México. J. Fish Biol. 44: 647-659.

Vega-Cendejas M.E., M. Hernández \& G. De La Cruz Agüero G. 1997. Los peces de la Reserva de la Biosfera de Celestún. CINVESTAV Mérida. PRONATURA. Península de Yucatán, México.

Whitfield A. K. 1999 Ichthyofaunal assemblages in estuaries: A South African case study. Rev. Fish. Biol. Fisheries 9: 151-186.

Zar, JH. 1984. Biostatistical Analysis, 2nd edition, Prentice Hall. New Jersey, USA.

\section{INTERNET REFERENCES}

Froese R, Pauly D. 2004. FishBase, version (09/2004). (Downloaded: Feb 24, 2005 www.fishbase.org). 
